\title{
Proposed Design and Assessment Methodology of a Wearable Device for Prevention and Performance Evaluation of Athletes
}

\author{
Athanasios Anastasiou, National Technical University of Athens, Greece \\ Angeliki Nikaki, Computer Solutions SA, Greece \\ Stavros Pitoglou, National Technical University of Athens, Greece \& Computer Solutions SA, Greece \\ (iD) https://orcid.org/0000-0002-5309-4683
}

Yiannis Koumpouros, University of West Attica, Greece*

(iD) https://orcid.org/0000-0001-6912-5475

\begin{abstract}
The paper presents the design approach of a low power stretchable wearable epidermal electronic device as part of an advanced athlete performance management framework to perform multi-parametric, multi-modal measurements of vital physiological parameters through the skin and via sweat analysis. It can be used to seamlessly monitor athletes to prevent serious health complications and injuries during training, sudden death, and complications related to overtraining. The proposed framework allows real-time physiological monitoring, tracking, prediction, and quantification of athletic performance and estimation of recovery time after an injury, an illness, or overtraining syndrome. It is based on low-cost and clean room-based fabrication techniques and interconnects and readout electronics based on commercially available off-the-shelf components and application-specific integrated circuits. A data analytics platform incorporates real-time monitoring to identify the susceptibility to sudden death, health complications, or injury for the athlete executing a personalized prediction model.
\end{abstract}

\section{KEYWORDS}

Athletes, mHealth, Mobile Health, Sudden Death, Wearable, Wearable Electronics

\section{INTRODUCTION}

Several epidemiological studies have shown that regular physical activity is associated with numerous health benefits, reducing the risk for cardiovascular disease (CVD) and other chronic diseases (e.g. obesity, diabetes mellitus, hypertension, depression) as well as improving morbidity, CVD and all-cause mortality (Lee et al., 2014). Exercise is an additional stressor to regular physical activity, a physical loading that enhances energy expenditure and dietary requirements, elevates body 
temperature and increases the demands on the cardiorespiratory system. Exercise may increase the risk of complications, ranging from musculoskeletal pain and strains to injury, fatigue, illness and hopefully rarely life-threatening disorders. Recent reports suggested a U-shaped relationship between exercise and mainly cardiovascular mortality (Lee et al., 2014). The incidence of sudden cardiac death (SCD) during sports activities varies significantly with age. Recent studies indicate that the rates of exercise-related SCD are around 1:50,000 Athlete-Years (AYs) in young athletes. Multiple studies have reported adverse effects of high intensity and high amount of training and competition load in athletes. $12.5 \%$ of the healthy London Marathon runners studied developed exercise-associated hyponatremia, a potentially fatal cause of the collapse (Eijsvogels et al., 2016). Athletes, especially those at the highest levels of their sport, tend to continue exercise and competition, even if they have physical complaints or limitations, increasing the need to use appropriate monitoring tools for safety reasons. The impact of exercise related to SCD and other health disorders is magnified to this specific group. Soft tissue injuries are the most common type of injury in athletes. These can lead to chronic pain, dysfunction, and reoccurrence and are often due to poor conditioning, dehydration, and overtraining. Current methods to assess fatigue, the anaerobic threshold during exertion, as well as the levels of electrolytes level which are essential for the prevention of arrhythmias and collapse, are either inadequate, currently not available or cannot provide real-time information. The vital key to reduce the above is the online monitoring of critical signs, electrolytes, oxygen saturation, $\mathrm{pH}$, glucose, cortisol and lactate levels during exercise.

Elite athletes and sports teams continuously search for methods and opportunities to gain a competitive advantage and improve athletic performance. Since the introduction of heart rate monitors in sports in the mid-70s, several sensors and activity trackers have been proposed to promote an active lifestyle and monitor and assist athletes during training (Merghani et al., 2017). The current state of the art in sport science clinical assessment of athletic performance involves using a plethora of systems and methodologies that are not portable and suitable for field deployment. Due to their bulkiness, they cause discomfort, add weight to the athletes or restrict their movement leading to unrealistic and biased test conditions (white coat effect). These involve traditional ECG (electrocardiography) monitors with large electrodes distributed across the Athlete's body, pulse oxymeters, ergospirometers and blood analysis for metabolite measurements.

Consequently, such approaches take the Athlete out of their regular training environment and routine and dictate the training conditions rather than facilitate them. In recent years, there have been several developments in activity monitoring. Motion sensors (pedometers, accelerometers, gyroscopes, magnetometers and GPS devices) are widely used by athletes to monitor posture, steps and physical activity, estimate energy requirements and expenditure, and measure speed and sport movement demands, modify training regimens and optimize performance. In sports medicine, several ICT (information and communication technologies) for health applications, platforms and devices have been used for the collection of physiological data in the attempt to maintain or improve Athlete's health and performance. Wearable sensors for monitoring athletes' heart rate, blood pressure, electrocardiogram and electromyogram, respiratory rhythm, body temperature, blood oxygen, glucose levels and galvanic skin response are available to monitor physiological responses to exercise (Kipps et al., 2011). The use of one such device (heart rate, body temperature and motion sensing) has been reported to reduce injuries by $60 \%$ (Jourdan et al., 2021). Popular commercially wearable electronic/ sensing devices for general health monitoring and sports applications (e.g. Fitbit and Samsung smartwatches) rely on standard electronics and sensors. The number of physiological parameters that can monitor is often limited to heart rate (typically using pulse oximetry) and motion (gyroscopes, accelerometers etc.). Such devices have not all been evaluated scientifically, and there are several parameters, as motion artifacts and environmental conditions, that may affect the quality of the data collected (Asif \& Harmon, 2017).

Consequently, their diagnostic and even general monitoring performance is limited and questionable. Moreover, motion artifacts are mainly due to the rigidity, large size and overall form 
factors of the devices, which prohibit conformal contact with the skin and their geometrical adaptation to induced mechanical stresses and motion during exercise. Nevertheless, such devices, combined with the widespread use of smartphones, have led to the development of wearable electronics.

However, recent advances in fabrication techniques, additive manufacturing, material science, microelectronics nano-materials and smart systems technology present new opportunities to expand the functionalities of such devices and enhance their diagnostic and monitoring capabilities. Traditional electronics, based on rigid silicon technologies, are associated with several intrinsic disadvantages. The inherent brittleness of inorganic semiconductors and stiffness of Si (silicon) wafer-based devices represent a significant issue when interfaced with tissues. Tissues are soft, and they undergo constant motion and deformation. Consequently, there is an inherent incompatibility between traditional electronics and the human body, leading to damages of both the tissue and the devices and unreliability in the obtained measurements. The development of flexible/stretchable wearable epidermal electronic systems with embedded sensors, sensor readout electronics and wireless connectivity for physiological monitoring of athletes can thus address the above challenges and needs. Measurements through the skin can provide a plethora of physiologically relevant information that can be used to monitor athletic performance and health. In addition to traditional measures of ECG and PPG (photoplethysmography), the chemical analysis of eccrine sweat can provide valuable information regarding an athlete's health by monitoring the concentrations of, e.g. vital electrolytes and metabolites. Such devices thus need to be flexible, stretchable, durable, biocompatible, lightweight, integrated with all the necessary sensors and electronics into a single device. Several such devices have recently been proposed in the literature. However, none of these has the range of sensing and functionalities targeted by the proposed solution and are needed for a holistic assessment of athletes (Li et al., 2016). The development of such devices is related to several challenges, including the development and integration of the necessary electrical, electrophysical, optical, and chemical sensors, the readout of sensor required electronics and wireless data transmission, and flexible/stretchable interconnect technology. Such smart devices and systems have the potential of generating a plethora of valuable information that can enhance human-to-physical world interaction and provide more significant insights into human physiology (Kuniharu et al., 2015).

\section{CONCEPT AND APPROACH}

The use of the proposed technologies commences when athletes, their coach and/or their physician are interested in monitoring and evaluating athletic performance during routine exercise and training, following injury and during or following rehabilitation, with potential future use in preventing sudden death in athletes (Benson et al., 2020; Seshadri et al., 2021; Gorski et al., 2021). The proposed wearable device will be able to measure in real-time a wide range of physiological parameters associated with both performance and health in a single, wearable, flexible/stretchable skin patch that the user can seamlessly wear. This will be performed non-invasively through the skin, either in the left hypochondriac region, the lower back in the lumbus, or below the armpit, depending on the sport. Such an approach is essential for athletes who do not accept devices that may hamper their movements during exercise and reduce their performance (Li et al., 2017).

The metabolism and physical state can be monitored via the recording of several parameters such as heart rate (via ECG and PPG) and tissue oxygen saturation (via PPG) and through these respiration and blood pressure via signal postprocessing to obtain the pulse transit time, skin hydration (via skin impedance measurements), measurement of various metabolites and ions such as lactate, glucose, $\mathrm{pH}, \mathrm{Na}^{+}, \mathrm{K}^{+}, \mathrm{Ca}^{2+} \mathrm{NH}_{4}^{+}, \mathrm{Cl}^{-}$and cortisol. Apart from pulse oximetry, these sensors can be based on electrodes, which will be post-processed electrochemically to develop electrochemical sensors (amperometry, potentiometry, impedimetric) and reduce contact imped of electrodes for ECG and bioimpedance. For pulse oximetry, commercially available LEDs and photodiodes will be used via interconnection technology. For heart rate measurements in addition to pulse oximetry, electrodes for 
Table 1. Parameters' indication for the athletes

\begin{tabular}{|c|c|}
\hline Lactate & $\begin{array}{l}\text { It is an indication of the pressure of tissue hypoxia and increases in glycogen } \\
\text { metabolism during exercise. Different blood lactate indices are used as predictors of } \\
\text { performance in athletes. }\end{array}$ \\
\hline Glucose & $\begin{array}{l}\text { It is the exclusive muscle fuel during strenuous exercise. Increased glucose utilization } \\
\text { may cause hypoglycaemic symptoms, like dizziness, confusion, weakness, seizure, loss } \\
\text { of consciousness, etc. }\end{array}$ \\
\hline $\mathrm{pH}$ & $\begin{array}{l}\text { The reduction of } \mathrm{pH} \text { has adverse effects on muscle function. Intense, prolonged } \\
\text { exercise may cause metabolic acidosis, leading to fatigue. }\end{array}$ \\
\hline $\mathrm{Na}^{+}$ & $\begin{array}{l}\text { Sweating during exercises causes excessive sodium loss. Symptoms of exercise-induced } \\
\text { hyponatremia are nausea, vomiting, headache, dizziness, etc. }\end{array}$ \\
\hline $\mathrm{K}^{+}$ & $\begin{array}{l}\text { Exercise may cause potassium deficiency with several symptoms such as nausea, } \\
\text { weakness, cramps, etc. }\end{array}$ \\
\hline $\mathrm{Ca}^{2}$ & $\begin{array}{l}\text { Sweat during exercises may cause calcium losses with symptoms like muscle cramps, } \\
\text { difficulty in concentration, muscle pain, etc. }\end{array}$ \\
\hline $\mathrm{NH}_{4}$ & $\begin{array}{l}\text { Ammonium ion increases during exercise, influencing the glycolytic energy } \\
\text { metabolism and triggering various neurotoxic consequences. }\end{array}$ \\
\hline ECG & $\begin{array}{l}\text { ECG may detect myocardial ischemia, supraventricular and ventricular arrhythmias or } \\
\text { conduction defects. }\end{array}$ \\
\hline Heart Rate & $\begin{array}{l}\text { Exercise helps increasing heart rate. The right training intensity should be chosen based } \\
\text { on the maximum heart rate, etc. }\end{array}$ \\
\hline Cortisol & $\begin{array}{l}\text { Intense exercise increases cortisol levels. Decreased concentration, muscle wasting, } \\
\text { weakness, etc., may be faced during overtraining. }\end{array}$ \\
\hline Skin Hydration & Dehydration during exercise may cause headaches, dizziness, disorientation, etc. \\
\hline Temperature & $\begin{array}{l}\text { Exercise may raise body temperature. Heat illness may be accompanied by headache, } \\
\text { fatigue, etc. }\end{array}$ \\
\hline $\mathrm{SpO}_{2}$ & Oxygen levels drop during exercise. \\
\hline Respiration & $\begin{array}{l}\text { Respiration rate is increased during exercise. Hyperventilation may cause chest pain, } \\
\text { weakness, arrhythmias, etc. }\end{array}$ \\
\hline Blood Pressure & $\begin{array}{l}\text { Blood pressure is increased during exercise. Dizziness, fatigue, etc., are common } \\
\text { symptoms of hypotension. }\end{array}$ \\
\hline
\end{tabular}

ECG as a more straightforward approach and organic electrochemical transistor (OECT) approaches can be developed. Temperature sensors, based on metallic filaments, can also be incorporated both as a vital physiological parameter and for their use in sensor calibration (the response of electrochemical sensors depends on temperature) (Lim et al., 2008). Electrophysiological, hydration and cardiovascular (CV) information can be obtained through direct contact of the device with the skin (via ECG and bioimpedance). PPG signal can be acquired through perylene and PDMS encapsulation. In contrast, sweat analysis initially directly on the skin and subsequently through sweat sampling microfluidic approaches will allow ionic and metabolite analysis (Lee et al., 2017). Consequently, the proposed solution can provide one of the most objective measures used to assess fatigue, the anaerobic threshold during exertion, and the electrolyte level, which is of high importance for preventing arrhythmias and collapse.

The collected data will be transmitted via Bluetooth to a portable device, analyzed in real-time, and further sent to the cloud, a system application server for further processing and storage, overcoming existing functional limitations relevant to real-time activity monitoring (Aughey, 2011). Data will be compared with the performance and medical history of the Athlete. Also, due to the covid-19 situation, we will be able to measure the pre-covid19 data and the after-covid19 data; thus, we can compare 
them and conclude on Athlete's performance (Jeong et al., 2021). This will allow the Athlete, their physician and/or their coach to assess their performance, to make informed decisions regarding their health, resting periods, hydration and intake of ions. The information generated via the proposed solution technologies will allow the actors to maintain a performance record and medical history.

Consequently, these technologies can allow the generation of a performance database for an athlete and/or a sports team, provide personalized training plans and informed decisions in the game plan of teams, and provide more significant insights into the physiology of professional athletes. Consent by the Athlete is granted, and ethical issues are addressed. The sports media can use professional bodies and an athlete's agent for recruiting purposes in the sports betting industry and the sports supplements industry. Consequently, the generation of such information has the potential of creating new opportunities in existing industries.

Several different approaches should be examined for the realization of the proposed flexible/ stretchable device:

1. In one approach, the sensor readout electronics could be developed using flexible printed circuits (FPCs) on polyimide substrates with a thickness of $\sim 125 \mu \mathrm{m}$. Incorporating the sensors on the same substrate is a simple natural extension to this technology, allowing flexible and (with the appropriate geometric design) stretchable sensors. This has a resolution of $\sim 50 \mu \mathrm{m}$. This is a low hanging fruit with the technology being already trialed and proven, allowing the end-users to manufacture, test, and use a complete device within three months. Early engagement of the end-users will enable the sensors and the electronics to be tested. It will provide feedback before the availability of the microfabricated device, which is the ultimate goal.

2. In a second approach, a custom low-cost fabrication technique with composite conductive polymers based on PDMS (polydimelthysiloxane), carbon-based and silver nanowire materials with a resolution of $\sim 250 \mu \mathrm{m}$ can be employed. Apart from the resolution, another disadvantage is related to the relatively thick PDMS substrate required, of a $\sim 1-2 \mathrm{~mm}$, due to a transfer printing step. This technology has the advantage of being inherently stretchable. Furthermore, this is a low-cost technology with a quick turnaround (a couple of days for all the interconnects and electrodes) with techniques suitable for large area manufacturing (stencil, screen printing, and laser machining). Prototypes have already been developed and are tested in a laboratory environment. The PDMS substrate allows the integration of the devices with microfluidics. Consequently, this technology can also enable early testing of the integration of the sensors with microfluidics.

3. In a third approach, flexible and stretchable organic electronic devices fabricated using photolithographic techniques with a five $\mu \mathrm{m}$ resolution can be examined as a high-end approach for higher density and greater miniaturization. Flexible/stretchable interconnects developed using the same approach can be used to integrate the recording electronics (microcontrollers, amplifiers, light-emitting diodes, photodetectors and other COTS components) designed in (1) within these devices. These devices can be initially fabricated on a flexible parylene substrate following current fabrication protocols. Stretchable substrates can also be examined to introduce stretchability and compatibility with PDMS-based microfluidics.

Consequently, someone can progressively optimize the patch fabrication going from the most straightforward but less wearable approach (FPC, PCB) to devices that can enhance first the wearability of the device and subsequently the integration. Typically, in multidisciplinary projects, the end-users are only involved towards the end of the project when output is available. In the meantime, they provide a superficial advisory role. Following the proposed approach allows end-users to be involved much earlier in the project. This will offer them a much more substantial input and advisory role regarding the operation, usability and accuracy of the device under realistic test conditions to allow optimization and targeted development in the other two solutions. In all three approaches, the incorporation of inductors for inductive charging/telemetry via high conductivity tracks should be explored. Each of 
these approaches is characterized by different risk factors, advantages and disadvantages and resolution leading to various form factors.

Consequently, this approach allows the minimization of risks and the examination and comparison of different systems with potentially different commercialization paths, costs and target users. The combination of the various techniques should be reviewed to leverage the advantages of each approach. The second and third approaches can be examined using two scenarios. The first involves the direct contact of all sensors to the skin. However, the repeated contact and friction with the skin and motion artefacts may prove detrimental to electrochemical sensor operation. Currently, flexible/stretchable multi-parametric sensing arrays still rely on traditional electronic components for signal processing, data acquisition, digitization and transmission. Advances have been made in flexible recording electronics, but these are currently limited to passive components, single transistors, or a handful of transistors to form simple amplifiers. Therefore, apart from fabricating the flexible/stretchable sensors and interconnects, researchers should also develop the necessary readout electronics using established techniques. Initially, a proof-of-concept system based on COTS components and FPCs with Bluetooth can be developed to interface with the fabricated flexible/stretchable sensing platforms. This approach is suitable for fast prototyping with quick turnaround, which will mainly serve as proof of concept of electronic readout topologies and allow robust testing of the developed multi-parametric flexible/stretchable sensing platforms, representative of the target applications testing environments. The disadvantage of this approach is that COTS components are general-purpose components not optimized for the target applications, leading to an overall large device footprint due to the need for multiple uses such as individual devices and high-power consumption. In a second approach, an application-specific integrated circuit (ASIC) can be developed for ultra-low power to minimize the device's overall footprint (Kim, 2016). This should include instrumentation amplifiers, Gm-C filters, trans-impedance amplifiers, voltage-current biasing subsystems, ADCs and DACs, etc., and will rely only on a small (e.g. Nordic) microcontroller for Bluetooth transmission or NFC (for scenarios where real-time monitoring is not necessary) for battery-less operation with a local commercial device (e.g. smartphone or tablet). NFC technology can also be exploited for the charging of embedded batteries. Silicon wafer thinning (mechanical and chemical, particularly with silicon-on-insulator CMOS technologies) can be explored to minimize the lateral ASIC dimension (Huang et al., 2012). COTS and ASIC based solutions can be incorporated initially in FPCs.

Figure 1. Overall Concept - Architecture
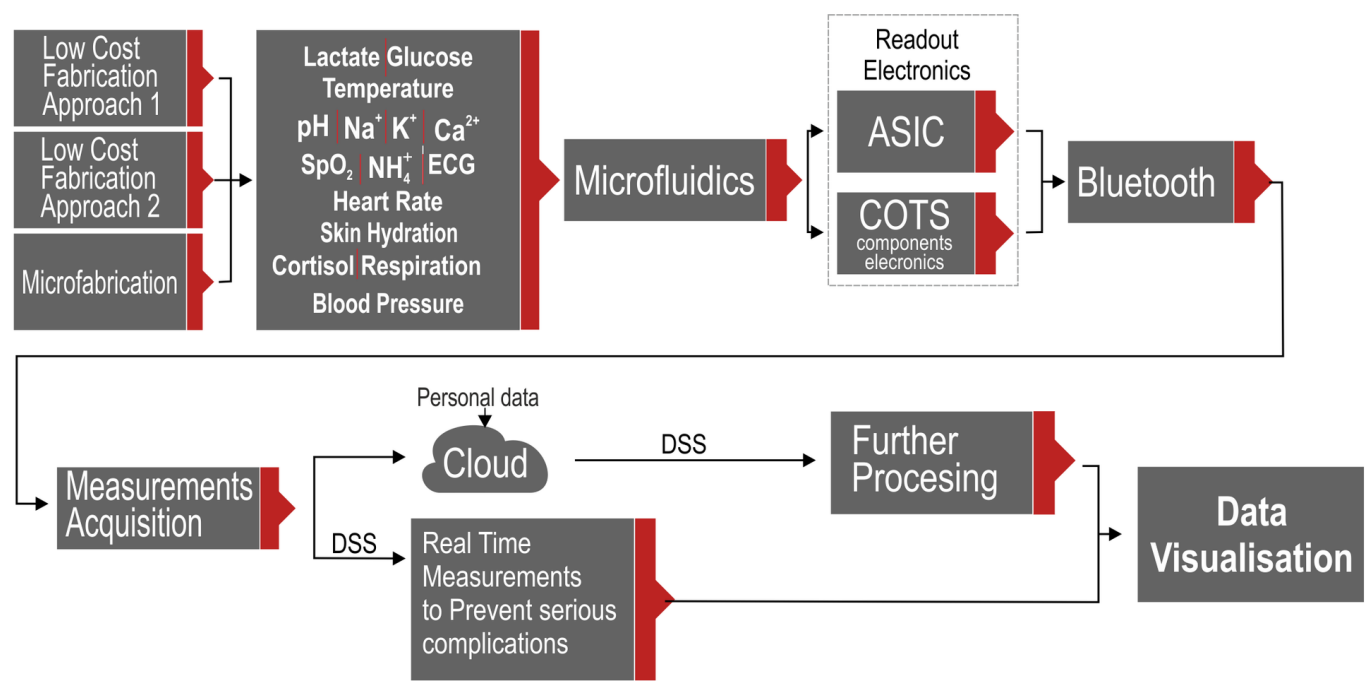


\section{Sensors}

Recent development in sensors promises excellent opportunities for monitoring athletes (Cheng et al., 2021). Detection of $\mathrm{pH}, \mathrm{Na}^{+}, \mathrm{K}^{+}, \mathrm{Ca}^{2+}$ and $\mathrm{NH}_{4}^{+}$can be performed via electrochemical potentiometric sensors. These are two electrode systems composed of a working electrode (WE) and a reference electrode (RE). A standard RE can be used for all potentiometric WEs, which should be gold, goldplated or conductive polymer electrodes with other electrodeposited membranes. For example, metalmetal oxide (MMO) electrodeposited membranes, such as IrOx, demonstrate super-Nernstian $\mathrm{pH}$ sensitivities $(>59 \mathrm{mV} / \mathrm{pH})$ and fast response times (Huang et al., 2012). Instead of applying a biasing potential to the RE, a differential measurement between RE and WEs with the solution biased via a third platinum electrode is used. Such differential measurements can minimize the effect of motion artefacts, drift, and other common-mode disturbances. Glucose and lactate can be monitored using electrochemical biosensors. Bio-functionalization of electrodes coated with conductive polymers can be performed to bind glucose and lactate oxidase enzymes onto the electrodes (Kim et al., 2017). The resulting electrode will be primarily used as the gate electrodes for OECTs (Lim et al., 2008). As an alternative, the same electrode functionalization strategy can modify the working electrode of a three electrodes cell and realize a standard amperometric biosensor. A cortisol immunosensor can be developed based on OECTs by forming a cortisol selective ion-blocking layer on top of the transistor channel (Kaushik et al., 2014). However, the transistor channel can also be modified with self-assembled monolayers (SAM) to immobilize cortisol antibodies on the surface. In contrast to all others proposed here, the disadvantage of such a sensor is that they are single-use sensors. However, the integration of multiples of the same sensors on the sensing patch may be used as a strategy to gain time interval information (Kim, 2011). Tissue oxygen saturation $\left(\mathrm{StO}_{2}\right)$ measurements can be obtained using techniques based on near-infrared spectroscopy, which have been tested in laboratory and clinical environments to be successful (Sankhala et al., 2018).

Temperature sensors can be based on meandering metal filament resistive sensor technology (Zhang, 2015). Moreover, long-lasting hydrogel and the PEDOT: PSS can provide a stable interface between the electrode and the skin. Similar methods can be used for the same purpose as the bioimpedance sensor for skin hydration measurements. The geometry of this should be optimized based on finite element simulations to enhance the sensitivity of the sensor to sweat in the epidermis and the sweat glands (Martín, 2017). For all targets, the proposed devices should have multiple dedicated sensors to allow averaging and provide redundancy in the case of sensor failure.

Figure 2. Sensitivity and response (inset) of an IrOx FPC-based pH sensor

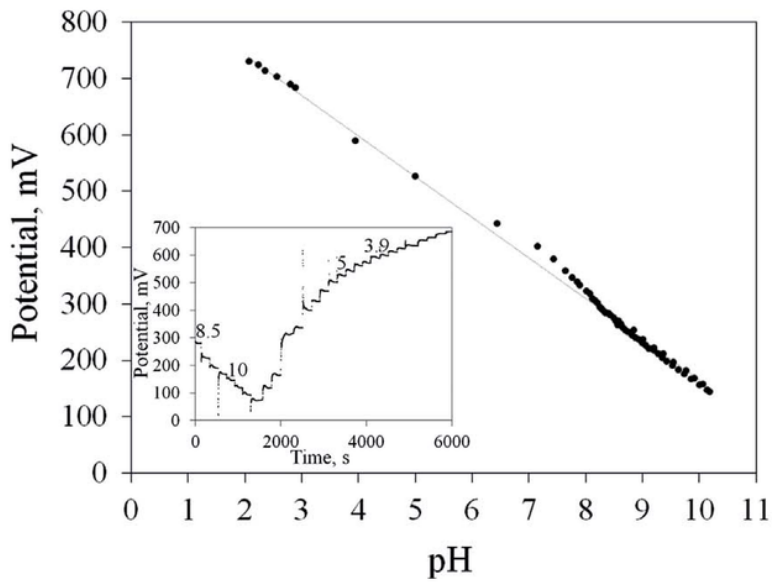


Figure 3. Response of a Na+ FPC-based sensor. Both measurements are obtained with FPC-based Res

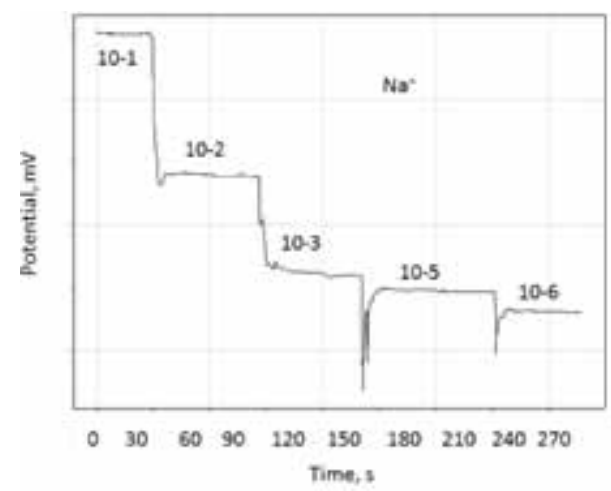

Figure 4. An FPC-based tetrapolar bioimpedance sensor optimized for interrogating specific tissue depth via optimization of its geometry and electric field properties
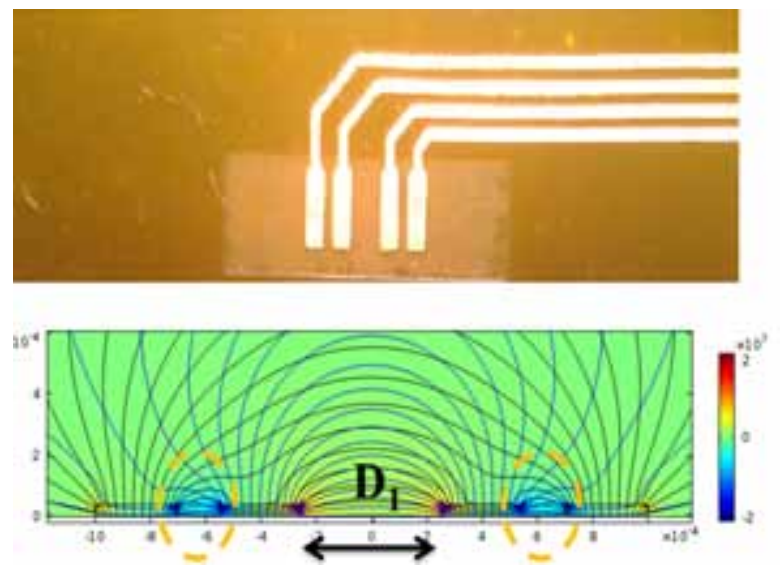

\section{Scenarios}

\section{Dizziness During Exercise: Differential Diagnosis}

Helen is 36 years old and has consistently run for over two years, $50 \mathrm{~min}$, five times a week. She ran her fourth marathon last Saturday. About 25 minutes into the run, she felt lightheaded and dizzy. Since she wanted to finish the run, she started to slow up and walked for about a mile. Then she drunk water and decided to continue running. She kept pushing into the dizziness and felt nausea, vomiting and like she needed gas and couldn't get it. She stopped and walked two times because she felt frail and faint. She breathed more deeply, drunk water and tried to remain calm, but things didn't get better. It could last a couple of minutes before she had to quit, seeking immediate medical care. She was given IV (intravenous) access, had her serum electrolytes checked and was diagnosed with hyponatremia due to fluid overload. She ended up consuming more fluids than she lost. That was an unpleasant and distressful experience. She is now scared about going running, and she is very disappointed about it. If Helen were wearing the proposed solution, she would prevent deterioration by avoiding further drinking water.

The proposed solution can help in the differential diagnosis of dizziness, nausea and weakness during exercise. Firstly, by measuring body temperature, if it is high, the diagnosis is heatstroke, while 
if it is normal, one must strongly consider the diagnosis of hyponatremia. The proposed solution must assess the electrolytes levels via sweat analysis (Kinnamon et al., 2017). The diagnosis can be confirmed by measuring the sodium levels and obtaining a result at or below $135 \mathrm{mmol} / \mathrm{L}$. Moreover, potassium and chloride levels are also low in hyponatremic athletes. If Helen was wearing the proposed solution and had seen the low electrolytes levels, she could have begun using oral salt supplements to correct for the losses. The proposed solution can also provide the glucose levels for the diagnosis of hypoglycemia, the blood pressure for the diagnosis of hypotension, the $\mathrm{SpO} 2$ levels for the diagnosis of exercise-induced asthma, and the heart rate (HR) and ECG monitoring for the detection of cardiac arrhythmias. Thus, the proposed solution is significant for preventing health disorders during exercise and ensuring the safety of exercise training.

\section{Performance Estimation After Summer Vacations}

Peter is an 18 years old soccer player and has progressed enough to sign a contract with a professional soccer club. He has been training very hard in the last two months, trying to be named to the firstteam squad. He was having two-a-day workouts, no rest during the week and is having a calorie deficit. During last week, he felt exhausted, weaker, had muscle soreness and stressed, was always irritated and had trouble sleeping. Although he worked hard, his performance was worsening, and his coach noticed that his resting and maximal heart rate was higher than usual. These are some signs of overtraining syndrome, common in athletes whose training is challenging to improve without recovery. Adequate rest is necessary, and the question is when and how can the Athlete return to play without complications. During the rest period, low-intensity exercise can help recovery. It is essential to create a custom training plan by lowering both the volume and the intensity of each workout. Thus, Peter will return to training wearing the proposed solution, which will monitor his vital signs during the workouts and keep his effort at a proper and safe moderate level. The proposed solution may track when he is not correctly recovering by monitoring his heart rate and blood pressure at rest and during each stage of his training session. Apart from the vital signs, the proposed solution will monitor the lactate levels, which will act as warning signals, telling Peter that his training should be reduced. A lactate curve will be created according to his lactate levels and exercise intensity.

Moreover, peak lactate levels will be a crucial marker for overtraining state. If his peak lactate levels increase during training, it is a sign that Peter can attain higher workloads. By monitoring both physiological and performance indices with the proposed solution, Peter can balance training stimulus and recovery and achieve his best-possible performance.

\section{Performance Estimation After Summer Vacations}

George is a 19 years old taekwondo athlete who competed at the international level and was committed to his challenging training schedule for the whole training year. After a hard-competitive season, he had three weeks of summer vacation and decided to rest and recover, avoiding hard training, diet and distress (Rong et al., 2021). Since all good things come to an end, he is now thinking about getting back on track and starting a new workout plan. International taekwondo athletes need to have moderate to high levels of cardiorespiratory fitness and high anaerobic power. Creating his training plan for the general preparation period, his coach started assessing his attributes to set realistic goals according to George's weaknesses and strengths. On the first day back to training, George wearing the proposed solution performed an incremental outdoor run on an all-weather track, and his vital signs and blood lactate levels were examined. After that, his coaches determined his lactate threshold (LT) and estimated his running velocity and HR at the LT. These are the appropriate methods for determining physiological variables associated with aerobic capacity (Vijayan et al., 2021). These indices were estimated easily by the proposed solution in the field, allowed George to be adequately trained according to his ability to ensure health, safety and effectiveness in performance. Training at the LT has been demonstrated to provide a significant stimulus for inducing endurance-oriented physiological adaptations. Thus, George wearing the proposed solution during his training will 
monitor his performance capability over time and progress to the next goal by increasing his training schedule's volume, intensity, and specificity. During the general preparation period, he needs to train smart and hard to avoid injury (Yu et al., 2017). The proposed solution will help his coach monitor and evaluate George's physical qualities in different periods of his annual season as far as the level of his physical fitness changes each season. (Peng et al., 2020).

\section{CONCLUSION}

The introduction of ICT integrated system solutions in Health and Sports Medicine is even more pronounced, where various actors at different involvement levels (athletes, coaches, sports enthusiasts) are intrinsically involved. Therefore, primary enabling requirements for ICT adoption are security, confidentiality, interoperability, scalability, and standards. The fundamental requirements for the adoption of an innovative technological solution into Athlete's habits and traditions are: i) the single solution is being implemented and tested within an official athlete's club environment; ii) the single solution is bringing a change in Athlete's performance monitoring, iii) the single solution is having an impact on Athlete's quality of life and ultimately health, iv) the single solution can transfer the experience to other organizations and systems. Those health-related ICT implementations that achieve the above fundamental requirements, in addition to assessment requirements, could scale up to the healthcare system. Assessment requirements are related to the measure of the impact of the ICT solution. A specific research study is needed to measure the impact of Athlete's health benefits, cost, social, and market dimensions. Thus, referring to the ICT solutions, the objectives of the solution should be defined alongside the adequate methodology to answer the purposes.

A thorough assessment methodology in three sequential steps is required for such a solution:

Step 1 - Acceptance assessment: The users' engagement in using the system and services reflects the degree of acceptance of the system and its functionalities by the target group. This is an essential prerequisite for the overall impact assessment of the project since evidence revealing the approval of the solution by the target population is fundamental to achieve any significant impact during and after the project's time course.

Step 2 - Benefits' assessment: The anticipated benefits from the use of the proposed service could be grouped in four role-based categories: (i) The benefits to Athlete's health, (ii) The benefits to Athlete's performance, (iii) The market's benefits, (iv) Indirect on Other Actors and/or Stakeholders.

Step 3 - Impact assessment: The measurement of the specific impacts of the proposed solution should be based on the Athlete's assessment results. These results can be derived from a list of measurable indicators of the outcomes of the offered service. In this way, the specific impacts expected from the system's use will be quantified objectively and credibly. 


\section{REFERENCES}

Asif, I. M., \& Harmon, K. G. (2017). Incidence and Etiology of Sudden Cardiac Death: New Updates for Athletic Departments. Sports Health, I(3), 268-279. doi:10.1177/1941738117694153 PMID:28452637

Aughey, R. J. (2011). Applications of GPS Technologies to Field Sports. International Journal of Sports Physiology and Performance, I(3), 295-310. doi:10.1123/ijspp.6.3.295 PMID:21911856

Cheng, Y., Wang, K., Xu, H., Li, T., Jin, Q., \& Cui, D. (2021). Recent developments in sensors for wearable device applications. Analytical and Bioanalytical Chemistry, 413(24), 6037-6057. doi:10.1007/s00216-02103602-2 PMID:34389877

Eijsvogels, T. M. H., Molossi, S., Lee, D., Emery, M. S., \& Thompson, P. D. (2016). Exercise at the Extremes: The Amount of Exercise to Reduce Cardiovascular Events. Journal of the American College of Cardiology, I(3), 316-329. doi:10.1016/j.jacc.2015.11.034 PMID:26796398

Gorski, M. A., Mimoto, S. M., Khare, V., Bhatkar, V., \& Combs, A. H. (2021). Real-Time Digital Biometric Monitoring during Elite Athletic Competition: System Feasibility with a Wearable Medical-Grade Sensor. Digital Biomarkers, 5(1), 37-43. doi:10.1159/000513222 PMID:33791447

Huang, X., Yeo, W. H., Liu, Y., \& Rogers, J. A. (2012). Epidermal Differential Impedance Sensor for Conformal Skin Hydration Monitoring. Biointerphases, I(4), 52. doi:10.1007/s13758-012-0052-8 PMID:22915327

Jeong, H., Lee, J. Y., Lee, K., Kang, Y. J., Kim, J. T., Avila, R., Tzavelis, A., Kim, J., Ryu, H., Kwak, S. S., Kim, J. U., Banks, A., Jang, H., Chang, J. K., Li, S., Mummidisetty, C. K., Park, Y., Nappi, S., Chun, K. S., \& Rogers, J. A. et al. (2021). Differential cardiopulmonary monitoring system for artifact-canceled physiological tracking of athletes, workers, and COVID-19 patients. Science Advances, 7(20), eabg3092. doi:10.1126/sciadv. abg3092 PMID:33980495

Jourdan, T., Debs, N., \& Frindel, C. (2021). The Contribution of Machine Learning in the Validation of Commercial Wearable Sensors for Gait Monitoring in Patients: A Systematic Review. Sensors (Basel), 21(14), 4808. doi:10.3390/s21144808 PMID:34300546

Kaushik, A., Vasudev, A., Arya, S. K., Pasha, S. K., \& Bhansali, S. (2014). Recent advances in cortisol sensing technologies for point-of-care application. Biosensors \& Bioelectronics, I(53), 499-512. doi:10.1016/j. bios.2013.09.060 PMID:24212052

Kim, D. H., Lu, N., Ma, R., Kim, Y.-S., Kim, R.-H., Wang, S., Wu, J., Won, S. M., Tao, H., Islam, A., Yu, K. J., Kim, T., Chowdhury, R., Ying, M., Xu, L., Li, M., Chung, H.-J., Keum, H., McCormick, M., \& Rogers, J. A. et al. (2011). Epidermal Electronics. Science, I(6044), 838-843. doi:10.1126/science.1206157 PMID:21836009

Kim, J., Campbell, A. S., Wang, J. (2017). Wearable non-invasive epidermal glucose sensors: A review. Talanta, 177, 163-170. 10.1016/j.talanta.2017.08.077

Kim, J., Salvatore, G. A., Araki, H., Chiarelli, A. M., Xie, Z., Banks, A., Sheng, X., Liu, Y., Lee, J. W., Jang, K.-I., Heo, S. Y., Cho, K., Luo, H., Zimmerman, B., Kim, J., Yan, L., Feng, X., Xu, S., Fabiani, M., \& Rogers, J. A. et al. (2016). Battery-free, stretchable optoelectronic systems for wireless optical characterization of the skin. Science Advances, I(8), 71. doi:10.1126/sciadv.1600418 PMID:27493994

Kinnamon, D., Ghanta, R., Lin, K. C., Muthukumar, S., \& Prasad, S. (2017). Portable biosensor for monitoring cortisol in low-volume perspired human sweat. Scientific Reports, I(1), 13-312. doi:10.1038/s41598-017-136847 PMID:29042582

Kipps, C., Sharma, S., \& Pedoe, D. T. (2011). The incidence of exercise-associated hyponatraemia in the London marathon. British Journal of Sports Medicine, I(1), 14-19. doi:10.1136/bjsm.2009.059535 PMID:19622524

Kuniharu, T., Wataru, H., Shingo, H., Takayuki, A., \& Seiji, A. (2015). Toward Flexible and Wearable HumanInteractive Health-Monitoring Devices. Advanced Healthcare Materials, I(4), 487-500. PMID:25425072

Lee, D., Pate, R. R., Lavie, C. J., Sui, X., Church, T. S., \& Blair, S. N. (2014). Leisure-Time Running Reduces All-Cause and Cardiovascular Mortality Risk. Journal of the American College of Cardiology, I(5), 472-481. doi:10.1016/j.jacc.2014.04.058 PMID:25082581 
Lee, H., Song, C., Hong, Y. S., Kim, M. S., Cho, H. R., Kang, T., Shin, K., Choi, S. H., Hyeon, T., \& Kim, D. H. (2017). Wearable/disposable sweat-based glucose monitoring device with multistage transdermal drug delivery module. Science Advances, I(3), 160-1314. doi:10.1126/sciadv.1601314 PMID:28345030

Li, Q., Zhang, L. N., Tao, X. M., \& Ding, X. (2017). Review of Flexible Temperature Sensing Networks for Wearable Physiological Monitoring. Advanced Healthcare Materials, I(6), 1601371. doi:10.1002/ adhm.201601371 PMID:28547895

Li, R. T., Kling, S. R., Salata, M. J., Cupp, S. A., Sheehan, J., \& Voos, J. E. (2016). Wearable Performance Devices in Sports Medicine. Sports Health, I(1), 74-78. doi:10.1177/1941738115616917 PMID:26733594

Lim, C. L., Byrne, C., \& Lee, J. K. (2008). Human thermoregulation and measurement of body temperature in exercise and clinical settings. Annals of the Academy of Medicine, Singapore, I(4), 347-353. PMID:18461221

Martín, A., Kim, J., Kurniawan, J. F., Sempionatto, J. R., Moreto, J. R., Tang, G., Campbell, A. S., Shin, A., Lee, M. Y., Liu, X., \& Wang, J. (2017). Epidermal Microfluidic Electrochemical Detection System: Enhanced Sweat Sampling and Metabolite Detection. ACS Sensors, I(12), 1860-1868. doi:10.1021/acssensors.7b00729 PMID:29152973

Merghani, A., Malhotra, A., \& Sharma, S. (2017). The U-shaped relationship between exercise and cardiac morbidity. Trends in Cardiovascular Medicine, I(3), 232-240. doi:10.1016/j.tcm.2015.06.005 PMID:26187713

Peng, B., Zhao, F., Ping, J., \& Ying, Y. (2020). Recent Advances in Nanomaterial-Enabled Wearable Sensors: Material Synthesis, Sensor Design, and Personal Health Monitoring. Small (Weinheim an der Bergstrasse, Germany), 16(44), e2002681.

Rong, G., Zheng, Y., \& Sawan, M. (2021). Energy Solutions for Wearable Sensors: A Review. Sensors (Basel), 21(11), 3806.

Sankhala, D., Muthukumar, S., \& Prasad, S. (2018). A Four-Channel Electrical Impedance Spectroscopy Module for Cortisol Biosensing in Sweat-Based Wearable Applications. SLAS Technology, I(6), 529-539. doi:10.1177/2472630318759257 PMID:29447045

Seshadri, D. R., Thom, M. L., Harlow, E. R., Gabbett, T. J., Geletka, B. J., Hsu, J. J., Drummond, C. K., Phelan, D. M., \& Voos, J. E. (2021). Wearable Technology and Analytics as a Complementary Toolkit to Optimize Workload and to Reduce Injury Burden. Frontiers in Sports and Active Living, 2, 630576.

Vijayan, V., Connolly, J. P., Condell, J., McKelvey, N., \& Gardiner, P. (2021). Review of Wearable Devices and Data Collection Considerations for Connected Health. Sensors (Basel), 21(16), 5589. doi:10.3390/s21165589 PMID:34451032

Yu, K. J., Yan, Z., Han, M., \& Rogers, J. A. (2017). Inorganic semiconducting materials for flexible and stretchable electronics. Flexible Electronics, I(1), 4. doi:10.1038/s41528-017-0003-z

Zhang, Y., Chad Webb, R., Luo, H., Xue, Y., Kurniawan, J., Cho, N. H., Krishnan, S., Li, Y., Huang, Y., \& Rogers, J. A. (2015). Theoretical and Experimental Studies of Epidermal Heat Flux Sensors for Measurements of Core Body Temperature. Advanced Healthcare Materials, I(1), 119-127. doi:10.1002/adhm.201500110 PMID:25953120 
Athanasios Anastasiou received his Bachelor degree in Electrical \& Electronic Engineering from the University of Newcastle Upon Tyne, in 2005. In 2006, he received his M.Sc. degree in Digital Signal \& Image Processing from the University of Central Lancashire. Likewise, in 2009 he obtained his second M.Sc. degree in Biomedical Engineering held by the University of Patras and the National Technical University of Athens (N.T.U.A.). In 2015, received his Phd degree in Biomedical engineering in the field of mobile health. Since 2011, he is a member of the Biomedical Engineering Laboratory of N.T.U.A.. His current research interests comprise Bioinformatics, Biosignal Processing, Digital Image Processing and technologies for assisted living. He is a Member of the Institute of Electrical and Electronics Engineers (IEEE) - EMBS Society and Computer Society, Member of the Institution of Engineering and Technology (IET) and a member of the Technical Chamber of Greece (TEE).

Stavros Pitoglou received his BSc in Physics from the University of Patras, Greece in 2000. He currently holds the Chief Technical Officer position in Computer Solutions SA, being responsible for the company's Research and Development activities. Since 2004 he participated as Project Director or Senior Project Manager in several large-scale projects in the fields of Health IT and Medical Informatics as well as Coordinator and Senior Researcher in Research/Innovation projects. His research interests are focused on issues of machine learning and artificial intelligence applications in the healthcare domain, distributed networks and data security-privacy. He is a PhD candidate in the Biomedical Engineering Laboratory, School of Electrical and Computer Engineering of the National Technical University of Athens, member of the Institute of Electrical and Electronics Engineers (IEEE) - EMBS Society, member of the European Association for the Study of Diabetes and a certified HL7 Control Specialist by HL7 International Organization.

Yiannis Koumpouros (BSc, MSc, MBA, PhD) is Ass. Professor in the University of West Attica, Department of Public and Community Health. Specialized in health informatics, e-health, m-health, telemedicine, assistive technologies, rehabilitation, users satisfaction assessment, and strategic management. Expert for the European Commission in the e-health and m-health sectors, and evaluator of numerous R\&D projects. Responsible for the WHODAS 2.0 scale cultural adaptation for the World Health Organization in Greece. Top-level managerial experience in the private and public healthcare sector (chairman of hospitals, member of the board of directors, business development and strategic management director, etc.). Chairman of several committees in the Ministry of Development. Regular member of the Consultative Technical Council (Ministry of Internal Public Administration and Decentralisation), etc. He has published more than 70 papers in reputed journals and conferences and has been serving as an editorial board member of repute. He has participated in more than 50 R\&D funded projects in the field of healthcare and ICTS. 